\title{
Radiology compared with xenon-133 scanning and bronchoscopic lobar sampling as methods for assessing regional lung function in patients with emphysema
}

\author{
C. E. BARTER ${ }^{1}, P$. HUGH - J ONES, J.W. LA W S, and \\ W. A. C R OS B IE \\ Chest Unit and Department of Radiology, King's College Hospital, London S.E.5
}

\begin{abstract}
Regional lung function was assessed by radiographic methods, by regional function studies using xenon-133 scans, and by lobar sampling with a mass spectrometer flow-meter at bronchoscopy in 12 patients who subsequently had bullae resected at operation. The information given by these three methods of regional assessment was subsequently compared with the findings at operation. When only one lobe was abnormal on the radiographs, these alone were adequate to locate the major site of the emphysema and the regional tests gave relatively little extra information. The xenon scan was sometimes helpful in assessing the state of the remaining lung, but this information could be deduced from the radiographs and overall lung function tests, especially the carbon monoxide transfer and mechanical measurements. Bronchoscopic sampling was helpful in determining whether the affected lobe was acting as a ventilated dead-space. When more than one lobe was affected the regional function tests supplemented the radiographs in defining the site of bullous change as well as locating dead space. Xenon scans, although widely employed for such preoperative assessments, added little to the topographical information obtained by careful radiology. The combination of radiology, lobar sampling, and overall function tests is recommended for assessing which emphysematous patients are likely to benefit from surgery.
\end{abstract}

Only a small proportion of patients with bullous emphysema are likely to benefit from operative removal of their bullae (Hugh-Jones, Ritchie, and Dollery, 1966 ; Pride, Hugh-Jones, O'Brien, and Smith, 1970). Recent work has established which tests of overall lung function are the most useful for preoperative selection (Pride et al., 1970) but the relative merits of different methods of regional study, which is necessary both for the accurate localization of bullae and to determine their ventilation and blood flow have not been systematically studied.

Of the regional function tests, bronchospirometry has been superseded either by radioactive gas methods or by lobar sampling at bronchoscopy because bronchospirometry itself only enables one whole lung to be compared with the other. Radioactive gas methods mainly provide topographical measurements of function (Ball, Stewart, Newsham, and Bates, 1962 ; Bentivoglio et al., 1963 ; Dollery and Gillam, 1963), whereas lobar sampling measures anatomical function on a

1Present address: The Repatriation General Hospital, Heidelberg West 3077, Victoria, Australia lobar, or occasionally segmental, basis (West, 1960; Hugh-Jones, 1967). But radiology also permits localization of bullae, and, with care, information about regional ventilation and blood flow can be inferred from radiographs, especially if whole lung tomographs and both inspiratory and expiratory films are taken.

The study we present here was made in order to compare the information which can be derived from radiology, which is freely available, with that obtained by radioactive xenon scans and by lobar sampling. This report concerns 12 patients who had been studied by all three methods prior to thoracotomy for bullous emphysema ; a comparison was made between each method and the subsequent findings at operation.

\section{METHODS}

RADIOLOGY Films were taken upright in both inspiration and expiration in the postero-anterior (PA) projection and on inspiration only in the lateral projection. Whole lung tomograms were taken in the A-P projection supine at $3 \mathrm{~cm}$ intervals usually at 6,9 , 
12,15 , and $18 \mathrm{~cm}$ from the table top. They were read independently of the regional lung function results by one of us (J. W. L.) and were subsequently compared with these results.

Reduction in blood flow was assessed from the radiological appearance of the pulmonary vascular bed (Laws and Heard, 1962). Occasionally, when extensive lung destruction was present, the remaining zones appeared hyperaemic but this was not recorded. Reduced ventilation to an area was not inferred from increased transradiancy alone unless the transradiant zone was surrounded by compressed lung or unless, on expiration, the bulla failed to contract or the transradiancy remained unaltered.

The localization of bullae or of areas of reduced ventilation or blood flow was recorded in two waystopographically for comparison with xenon scanning, and anatomically for comparison with bronchoscopic sampling. For the former each lung field was divided into three approximately equal zones corresponding with the zones of the xenon study. The upper zone was the lung field above the sternal angle, the middle from the sternal angle down to halfway to the diaphragm, and the lower zone was below this line. Each zone was assessed in the mid-clavicular line for ventilation and blood flow. For the anatomical assessment the PA, lateral, and tomographic films were compared to determine which lobes were chiefly affected by emphysematous change.

RADIOACTIVE GAS SCANS The scanning was done at the Medical Research Council's Cyclotron Unit, Hammersmith Hospital, using two pairs of vertically moving counters as described by Dollery and Gillam (1963). All patients were seated upright for the scans.

To measure perfusion xenon-133 in solution was injected by an intravenous catheter whose tip lay in the superior vena cava and the counters were moved up the lung during breath-holding. Ventilation was measured both from the regional count-rate after a single inhalation of air containing xenon gas, and also from the time taken for the count-rate to reach its final value after rebreathing the xenon or from the time for this final count to halve during the subsequent rebreathing of air.

The counts obtained from each side of the chest were divided into 12 (inch-wide) bands, four above and eight below the sternal angle. These bands were then grouped into three equal zones on both sides of the chest and the percentages of ventilation and of perfusion for each zone were compared with the ranges derived from normal subjects using the same technique (Fig. 1a).

LOBAR SAMPLING A small flow-meter (West, 1960) was positioned sequentially into each lobar bronchus during bronchoscopy under local anaesthetic with the patient supine. An oesophageal balloon connected to a pressure transducer provided a record of the intrapleural pressure swings during the lobar flow recordings, so that the onset of inspiration was known. The lobar tidal volumes for comparable breaths were then obtained from integration of the argon flow signals recorded by a respiratory mass spectrometer (Fowler and Hugh-Jones, 1957). Cafibration curves for the flow-meter were obtain $\mathrm{d}$ during each study and these allowed the flow-metr to be corrected for alinearity (Hutchison and Smin, 1971). The percentages of the tidal volume going $\mathscr{H}_{0}$ each lobe were then related to the findings from normal subjects (McGrath and Hugh-Jones, 1963) . . depicted in Fig. $1 \mathrm{~b}$.

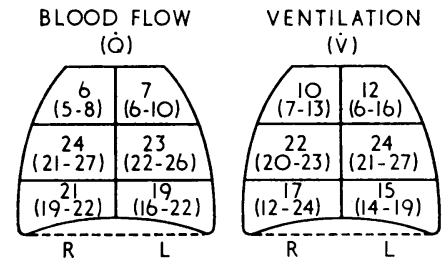

(a) XENON MEASUREMENTS

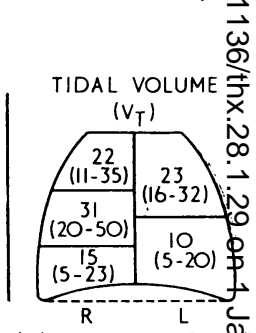

(b) LOBAR SAMPLI
FIG. 1. Normal values for mean percentage distribution blood-flow and ventilation (range in parentheses) to the six zones of the lung as measured from xenon scans (a); and for the lobar tidal volumes from the bronchoscopic measurements $(b)$. Note: $R$ and $L$ show right and left lung, respectively; this configuration is the same in all subsequent diagrams.

In addition, for each lobe, the argon supply was. cut off after the lobar ventilation had been measur $\overrightarrow{\mathrm{g}^{\mathrm{d}}}$ so that the flow-meter became simply a sampling tube for the mass spectrometer, and the changing. carbon dioxide and oxygen concentrations we recorded. This enabled us to detect impaired perfusion of a lobe relative to its ventilation, or asynchronous ventilation from obstruction to air-flof. The latter causes a given lobe to ventilate slighty out of phase with the rest of the lung, because gif a different time-constant, so that there is a shit period when it is still inhaling while the rest of the lung is exhaling. This is seen as a small notch Ð $\mathrm{dr}$ 'pip' (the ' $A$ ' pattern) at the start of the rise in $C \widehat{Q}_{2}$ (or fall in $\mathrm{O}_{2}$ ) concentration as the alveolar plateă is approached (West and Hugh-Jones, 1959; HughJones and West, 1960). Reduced perfusion relative to gas flow produces a 'pip' after the lowered alveolgr plateau itself (the ' $B$ ' pattern) as gas of the concentration in the dead-space which has come from th. rest of the lung enters the lobe before fresh air.

In two cases ( 2 and 3 ) exact figures for percentages. of distribution of lobar ventilation have not been given. This is because ventilation to some lobes was so high that the flow tracings 'squared off', whiक्क्र meant that the tidal volume to these lobes coutd not be fully measured. In these same patients them was almost no recordable ventilation in the lobs designated as being abnormal (Figs 6 and 8).

SELECTION OF PATIENTS The 12 patients studied formed part of a larger group referred for assesment of regional lung function following discovery. 


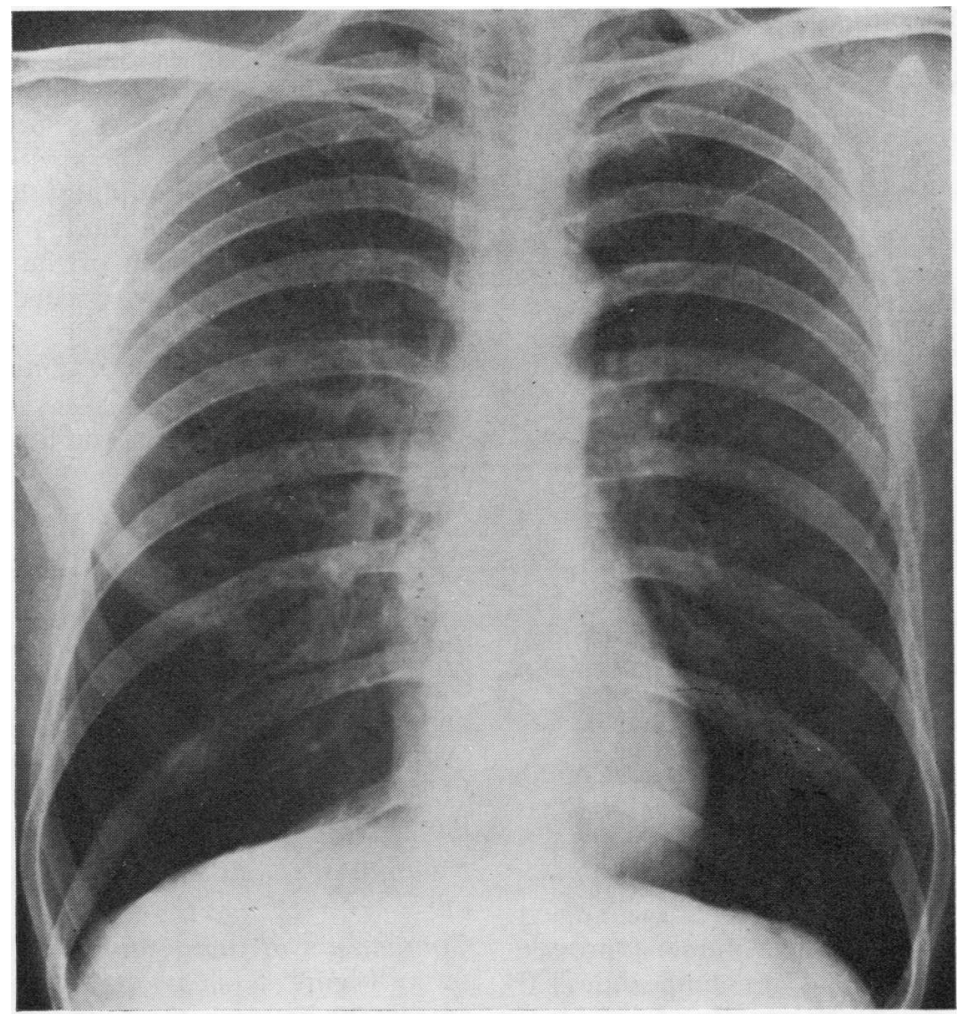

(a)

of localized radiological translucency. They were selected because all of them subsequently underwent thoracotomy and the surgical findings are available for comparison with the preoperative tests. There were 11 men and one woman, whose ages ranged from 38 to 62 years.

\section{PRESENTATION OF RESULTS}

The results of routine tests of overall lung function are only briefly presented, although they were done on all patients and have been reported previously (Pride et al., 1970 ; Pride, Barter and Hugh-Jones, 1972). The forced expiratory volume in one second $\left(\mathrm{FEV}_{1}\right)$, the transfer factor for carbon monoxide (TF), and the arterial oxygen tension $\left(\mathrm{PaO}_{2}\right)$ are given for each case. The radiographic results are presented in two forms to be compared with the regional function tests: first, zonal for comparison with the xenon scans and, secondly, in lobar form for comparison with the bronchoscopy sampling. To illustrate the method details are shown for one patient (case 7) whose chest radiographs are reproduced (Fig. 2) and the

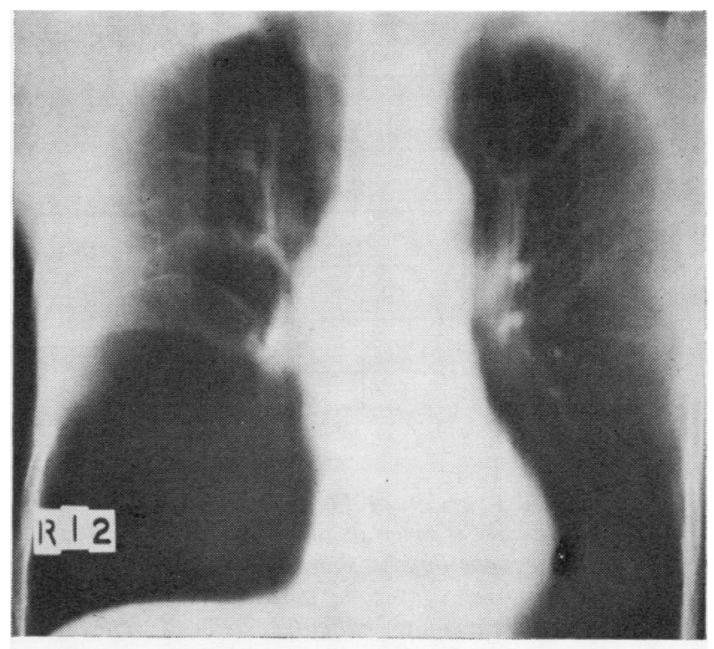

(b)

FIG. 2. Chest radiographs of patient 7 before operation: (a) postero-anterior view and (b) $12 \mathrm{~cm}$ tomograph. 
comparison of the radiologist's assessment of local function with the independent results of the tests is shown graphically (Fig. 3).

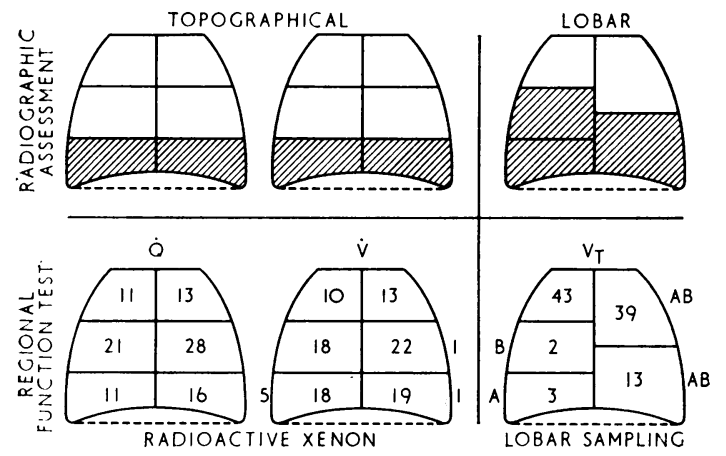

FIG. 3. Example of method of comparing independent radiographic assessment with regional function tests (patient 7).

For the purpose of general presentation of all the results a simplified diagrammatic method has been used (Fig. 4). Only the side operated on is presented for both radiological and regional function tests. Within each lobe or zone appear perfusion, ventilation or tidal volume expressed as a percentage of the total for both lungs. If these percentages are abnormally low close shading appears within that zone. Prolonged times of gas washout are shown beside the appro-

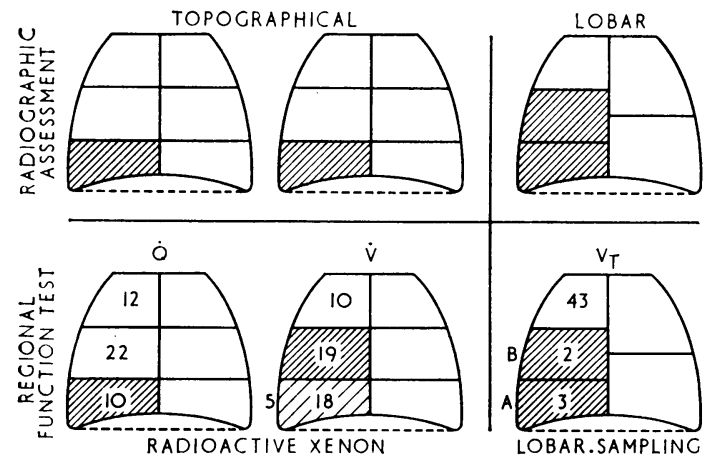

FIG. 4. Simplified version of Fig. 3, in which assessment of operated side only is given as an example of the method used in all subsequent figures showing such a comparison. (Close shading of relevant zone indicates reduced values on all tests; open shading indicates reduced ventilation values on one test only; figures outside diagram indicate prolonged time of gas washout in minutes; letters $A$ or $B$ represent corresponding patterns on mass spectrometer trace-see text.) priate zone of the xenon-133 scan in minutes. If the time of gas washout is prolonged and the single-breath index of ventilation is within norma稏 limits then that zone has been only partiall shaded. If ' $A$ ' pips or ' $B$ ' pips were found in the lobe during lobar sampling then these are diso played beside the relevant lobes in the loba $\overrightarrow{0}$ sampling diagram. Each of the 12 cases is placed into one or two groups depending on the radiow logical assessment:

Group 1. Only one lobe abnormal in the radio graphs.

Group 2. More than one lobe abnormal in the radiographs (either bullous or non-bullous emphy sema).

\section{RESULTS}

GROUP 1 (only one lobe abnormal radiologically CASE 1 (A.R., male, aged 55) Smoked 30 cigarettes/day for years, with bronchitis over the last 10 or more years. Dyspnoea for one year. FEV $_{1} 60 \%$ of predicted; TF $56 \%$ predicted $\mathrm{PaO}_{2} 81 \mathrm{mmHg}$.

Radiologist's opinion Large bulla in left lowe lobe.

Comparison of radiography and regional function tests Figure 5 shows that there was complete agreement between the topographical radiologicap and xenon findings and between the radiologica and lobar bronchoscopic findings.

Thoracotomy A large solitary bulla was found in the left lower lobe. The rest of that lobe and the whole of the left upper lobe appeared normal-o The bulla was excised.

CASE 2 (L.A., female, aged 54 years) Well untiB two years ago when she had a vague onset oR periodic breathlessness accompanied by bron?

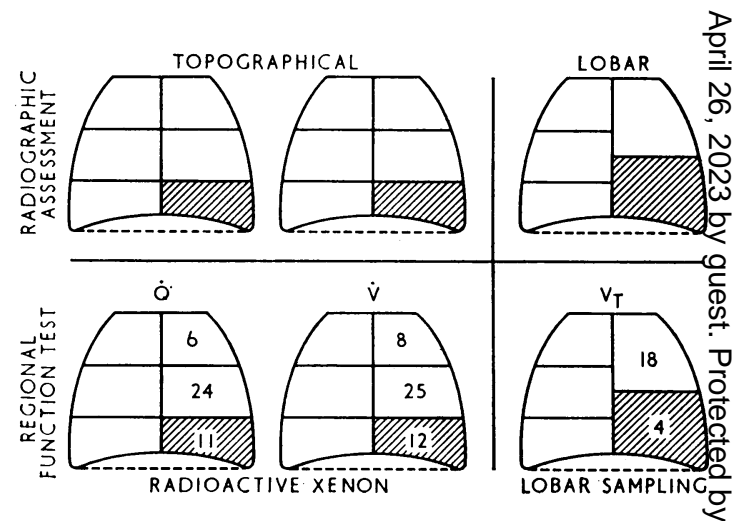

FIG. 5. Results for patient 1 . 


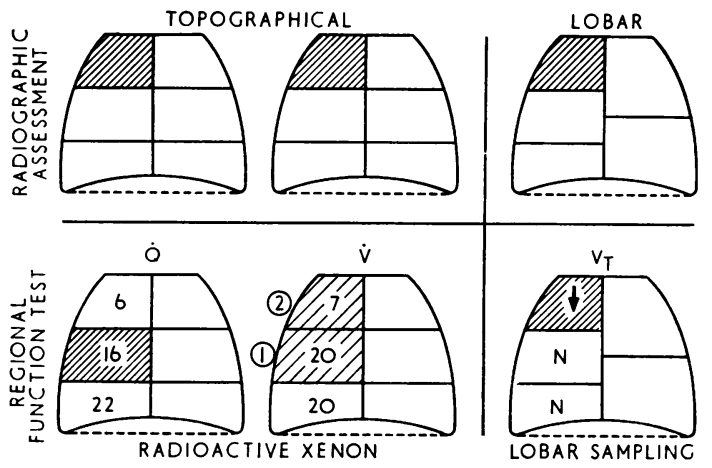

FIG. 6. Results for patient 2. (In lobar sampling $N$ means approximately normal, while $\downarrow$ means much reduced, ventilation.)

chitis. Life-time cigarette smoker. $\mathrm{FEV}_{1} 32 \%$ of predicted; TF $35 \%$; $\mathrm{PaO}_{2} 78 \mathrm{mmHg}$.

Radiologist's opinion Bullae confined to right upper lobe.

Comparison of radiography and regional function test Figure 6 shows that the radiological assess- ments agreed essentially with both the lobar and xenon results. That the xenon washout from both the upper and middle zones was abnormal is difficult to interpret, relative to the anatomical radiological and lobar findings, since these two zones include middle and lower lobes.

Thoracotomy There were multiple bullae in the apical and posterior segments of the right upper lobe. This lobe was the most abnormal, though there was also some emphysematous change in both the right middle and lower lobes.

CASE 3 (D.L., male, aged 38 years) Moderate effort dyspnoea for several years; not much bronchitis. Smoked over 20 cigarettes/day. FEV $136 \%$ of predicted; TF $48 \%$ predicted ; $\mathrm{PaO}_{2} 72 \mathrm{mmHg}$. Radiologist's opinion Large bulla in apical segment of left lower lobe compressing the left upper lobe.

Comparison of radiography and regional function tests Superficial assessment of the $x$-ray suggests that the bulla is situated in the left upper lobe (Fig. 7). Both physicians and surgeons had assumed this to be the case and were puzzled by the bronchoscopic findings. In fact, the radiolo-

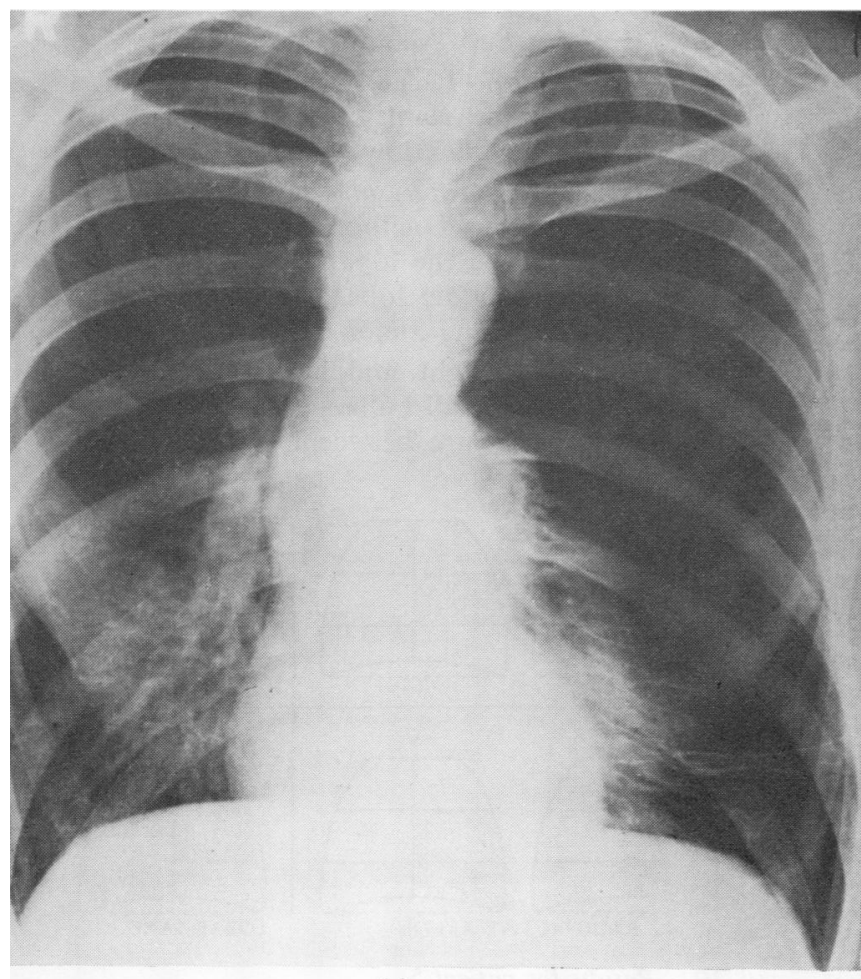

(a)

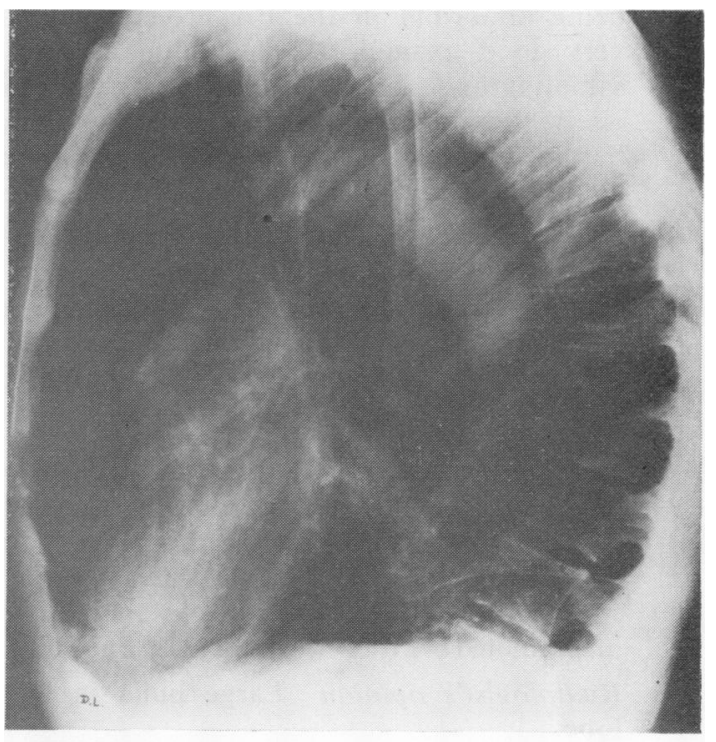

(b)

FIG. 7. PA and left lateral radiographs of patient 3 showing bulla in left lung which had been thought to arise in the left upper lobe until the radiologist and the bronchoscopy findings, independently, suggested that it arose in the left lower lobe, a suggestion which was confirmed at subsequent operation. 
gist, by careful scrutiny of the lateral radiographs and tomographs independently, came to the conclusion that the bulla arose in the apical segment of the left lower lobe and his anatomical findings agreed precisely with the lobar sampling (Fig. 8). The left upper lobe is not shaded in Fig. 8 by the radiologist because he thought that the lobe

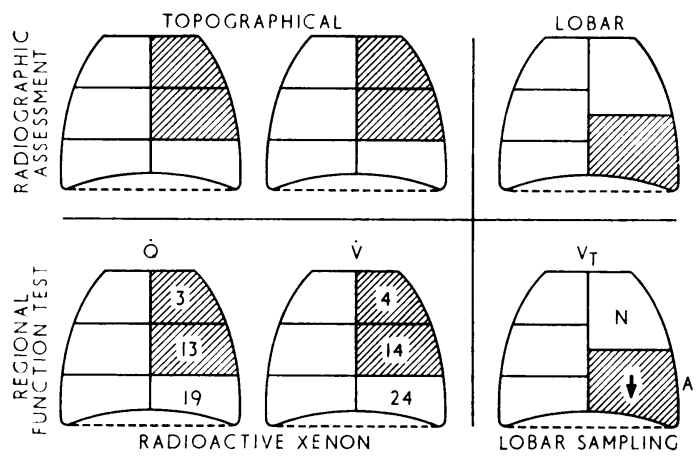

FIG. 8. Results for patient 3 .

was essentially normal, merely compressed (in contrast to the right lower lobe in case 7 below). On the other hand, the left upper zone is shaded as being abnormal in the PA projection. This is a very good example of the value of both lobar sampling and skilled radiography in locating precisely the correct anatomical position of a bulla before operation in the face of apparently contradictory topographical findings both from the xenon-133 and the $\mathrm{PA}$ radiograph alone.

Thoracotomy Large bulla in apical segment of the left lower lobe compressing left upper lobe. Emphysema in lateral segment of left lower lobe. Rest of left lower lobe and whole of left upper lobe appeared to be normal (the bulla was resected).

CASE 4 (A.G., male, aged 46 years) Classical bronchial asthma since age 25 years. Continual effort dyspnoea for three years. Chronic bronchitis since age 30. Pipe smoker only. FEV $33 \%$ of predicted; TF $55 \%$ predicted ; $\mathrm{PaO}_{2} 69 \mathrm{mmHg}$. Radiologist's opinion Large bulla in left lower lobe.

Comparison of radiography and regional function tests The radiologist, having sited the lesion as a bulla in the left lower lobe, agrees with the lobar sampling but he found it difficult to be sure whether the left mid zone on the PA view was abnormal in his assessment for comparison with the regional xenon findings because the bulla was partially masked by the overlying normal lefo upper lobe. This zone is partially shaded irp Fig. 9. The xenon results reflect this state o affairs.

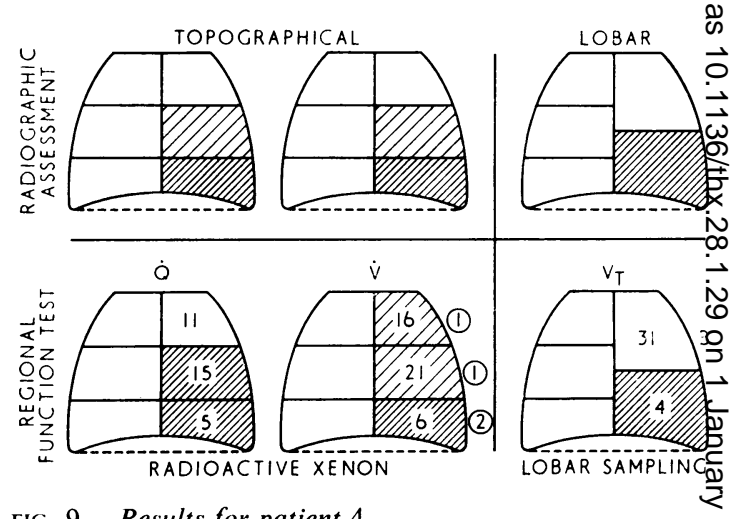

FIG. 9. Results for patient 4.

Thoracotomy There was a large bulla in the left lower lobe and the left upper lobe appeare $\$$ normal.

CAS $\equiv 5$ (E.S., male, aged 50) Bronchitis for 1 years. Effort dyspnoea for four years. Twent cigarettes/day since age $14 . \mathrm{FEV}_{1} 31 \%$ of pre dicted; TF $38 \%$; $\mathrm{PaO}_{2} 53 \mathrm{mmHg}$.

Radiologist's opinion Large bulla in right middle lobe compressing the right upper lobe medialle and the right lower lobe downwards.

Comparison of radiography and regional functio tests (Fig. 10) All three methods essentially agrew but the radiology and the lobar sampling site the bulla in the right middle lobe, a point not accue. rately portrayed by the xenon.

Thoracotomy The right middle lobe was com pletely replaced by small bullae and a large bulls was found in the fissure between the right uppes

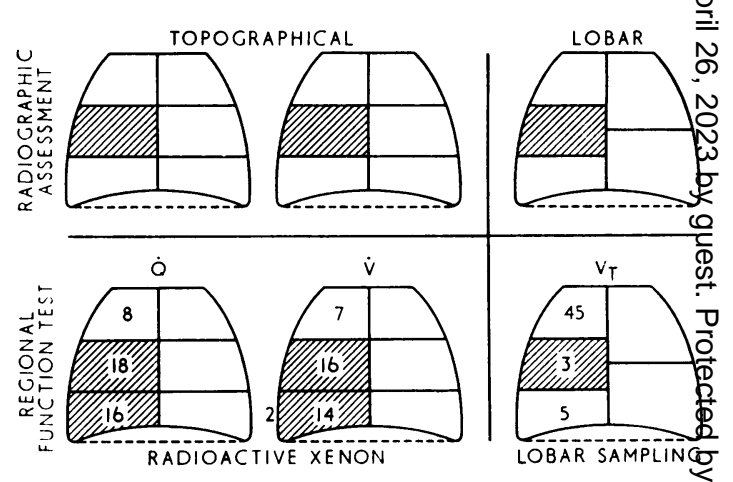

FIG. 10. Results for patient 5 . 


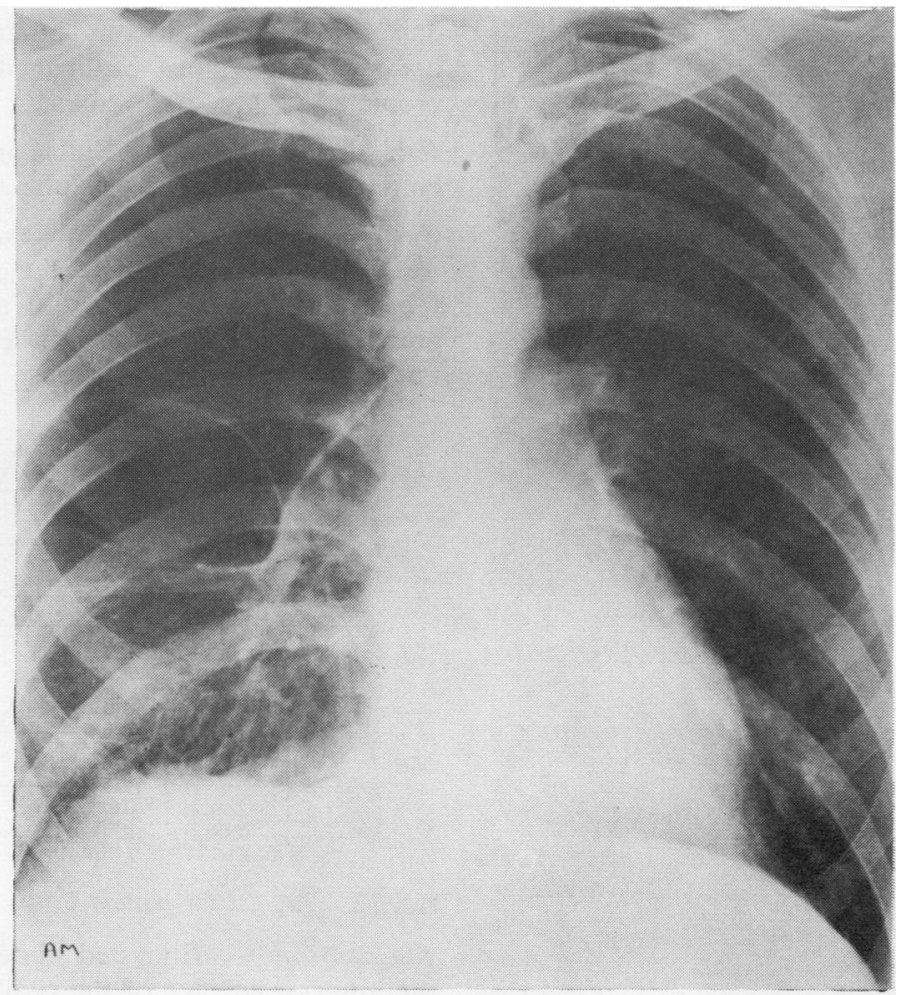

(a)

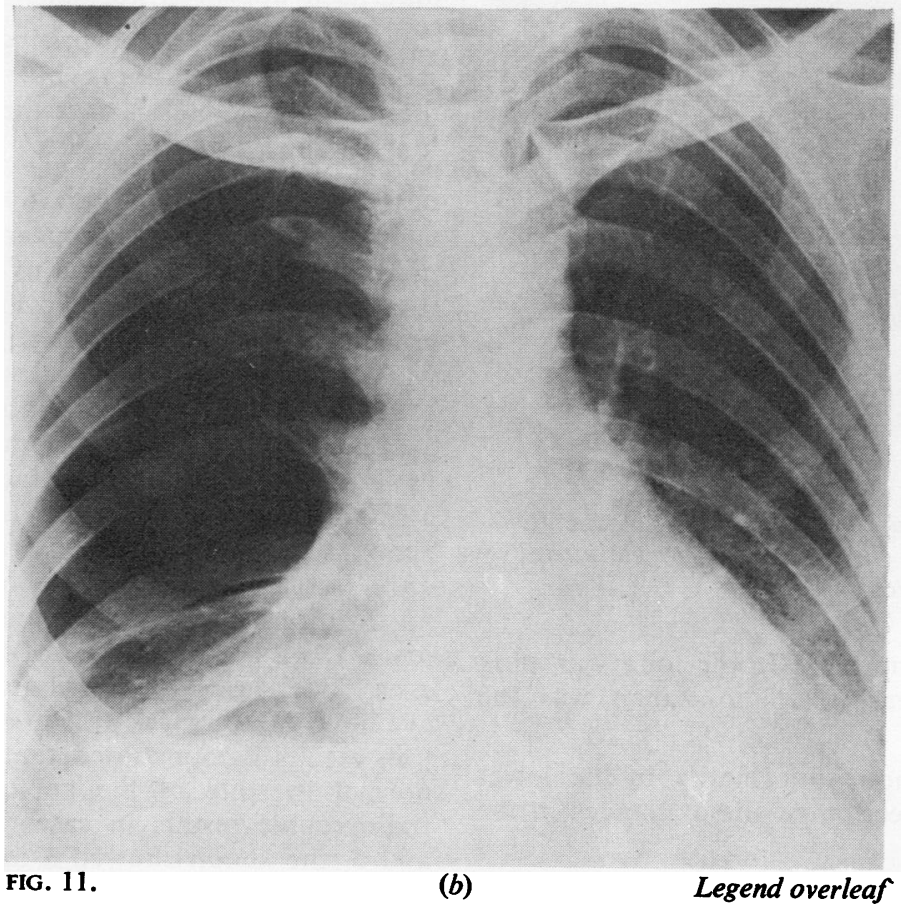




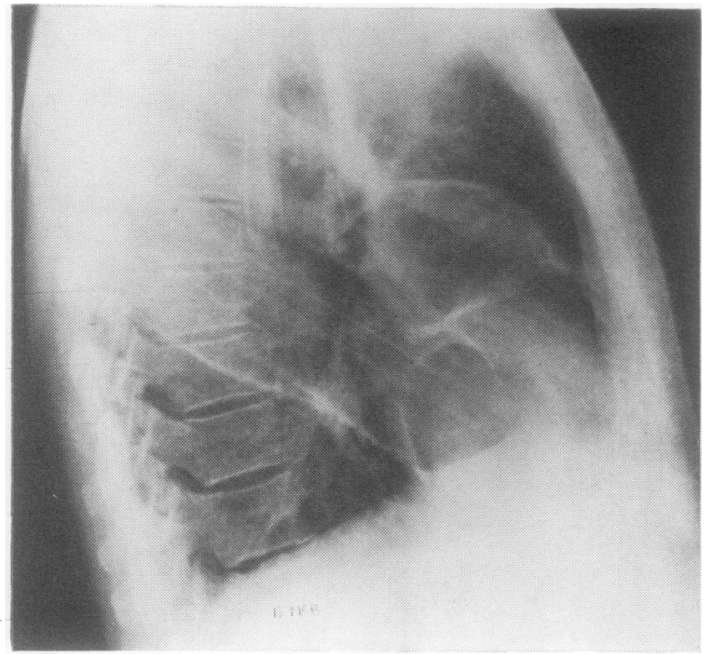

(c)

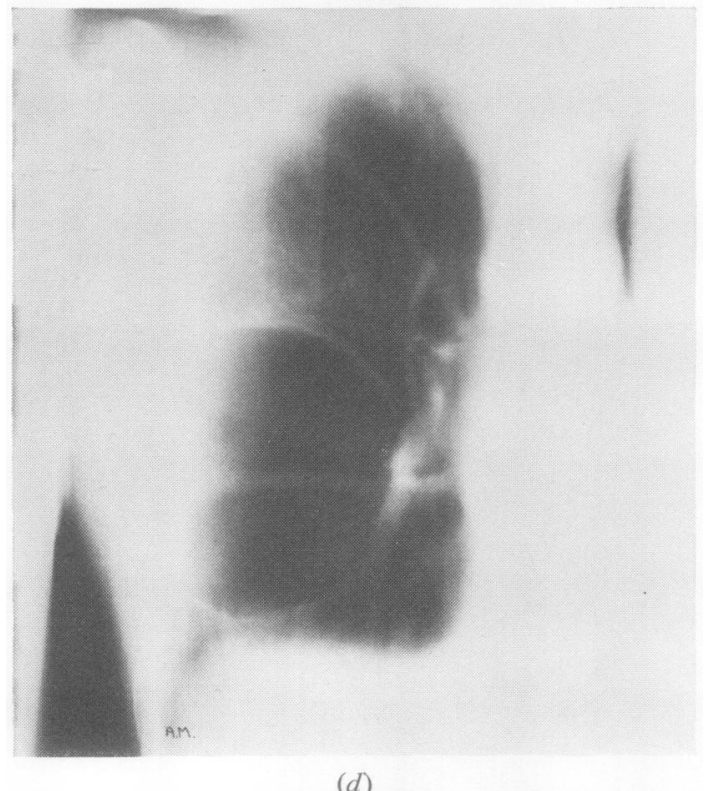

FIG. 11. Inspiratory (a) and expiratory (b) $P A$ radiographs of patient 6 with the corresponding right lateral (c) and relevant tomographic cut $(d)$.

and lower lobes. The right lower lobe was pink in colour and slower to collapse than was the right upper lobe.

Commert The xenon abnormality in the lower zones may be a reflection of these changes in the lower lobes.

CASE 6 (A.McB., male, aged 39) Two previous episodes of spontaneous pneumothorax, but no history of dyspnoea or bronchitis. $\mathrm{FEV}_{1} 68 \%$ of predicted ; TF $55 \%$ predicted ; $\mathrm{PaO}_{2} 94 \mathrm{mmHg}$.

Radiologist's opinion Bullae in right middle lobe compressing right lower lobe (Fig. 11).

Comparison of radiography and regional function $\vec{P}$ tests (Fig. 12) Whereas the radiology and the lobar sampling are again in agreement, there is complete disagreement between the xenon scan $\vec{x}$ and these other two methods.

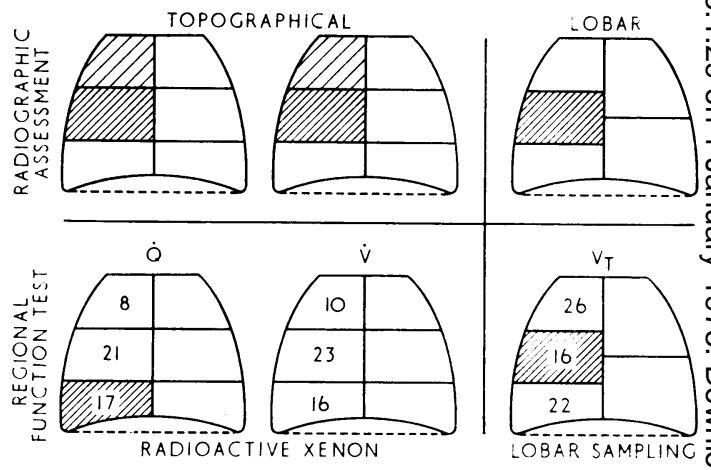

FIG. 12. Results for patient 6.

Thoracotomy Encysted pneumothoraces over the anterior surface of the right lung, although the lung itself appeared normal.

Comment This was surprising in relation to the radiological appearances but was supported by the normal ventilation over the whole of the righte lung as shown by the xenon.

COMMENTS ON CASES 1 TO 6 It is evident that where only one lobe appears to be abnormals radiologically, careful scrutiny of the radiographs (using inspiratory and expiratory films and wholeo lung tomograms) predicts well what the surgeon will find at operation. This was true in all these cases except case 6 , which, in our experience, was a most unusual case anyway. The other two methods only occasionally add information nof available from the radiographs. The weakness of xenon scanning is that it is purely topographicab and can be misleading about the anatomicalo localization of bullae in regional emphysema (case 3 is a good example of this). It does, how ever, uccasionally contribute information noto available radiologically: for example, the ventilation results demonstrated that the right lung was normal in spite of the apparently contradictorycD radiographic results in case 6 . Again, in cases $2 \frac{2}{6}$ and 5 , the xenon showed a more extensive lesion than that suggested by the PA radiographs and 
this xenon result was supported by the subsequent operation findings. The lobar sampling is as good as the radiograph in accurately localizing the site of the lesion anatomically. It, too, occasionally adds information not available from the radiographs ; for example, it can detect the infrequent but important occurrence of ventilation of a bulla causing greatly increased physiological dead space.

GROUP 2 (where more than one lobe was affected by emphysema, bullous or otherwise, on the radiograph)

CASE 7 (A.J., male, aged 58 years) Following three attacks of pneumonia, he developed rapidly progressive dyspnoea. No bronchitis previously. FEF $_{1} 46 \%$ of predicted; TF $52 \% ; \mathrm{PaO}_{2} 53$ $\mathrm{mmHg}$.

Radiologist's opinion (Fig. 2) Large bulla in right middle lobe compressing right lower lobe with emphysematous change, as judged by vasculature in right lower lobe.

Comparison of radiology and regional function tests (Fig. 4) Good agreement by all three methods.

Thoracotomy Large bulla in the fissure between the right middle lobe and right lower lobe, but this bulla was being ventilated from the right lower lobe. The right upper lobe was normal.

Comment It is of interest that the xenon scan showed a greatly prolonged time of gas wash-out over the right lower zone and equally that there was an ' $A$ ' pip at bronchoscopy in the right lower lobe, which was the lobe ventilating the bulla as shown by operation.

CASE 8 (J.H., male, aged 45) Attacks of winter bronchitis since childhood. Severe effort dyspnoea for five years. Smoked 30 to 40 cigarettes/day for over 30 years. $\mathrm{FEV}_{1} 29 \%$ of predicted; TF $45 \%$ predicted; $\mathrm{PaO}_{2} 74 \mathrm{mmHg}$.

Radiologist's opinion Reduced vascularity in the right middle and lower lobes. No localized bullae or air-trapping.

Comparison of radiology and regional function tests Essentially, there is agreement by all three methods (Fig. 13).

Thoracotomy Diaphragmatic surface of right lower lobe replaced by small bullae. This lobe was emphysematous except in its apical segment. It was resected.

Comment Again there was a prolonged time of gas wash-out in the xenon scan in the lateral zone

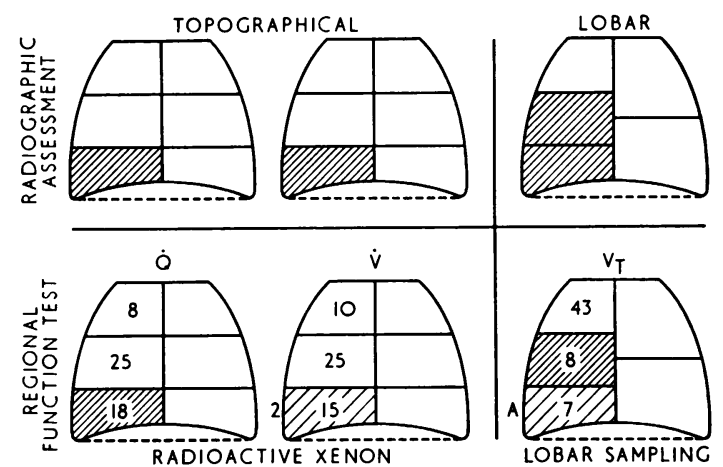

FIG. 13. Results for patient 8 .

and an ' $A$ ' pip present in the abnormal lobe at bronchoscopic sampling, even though the lobar tidal volume was within normal limits.

CASE 9 (C.V., male, aged 62 years) Minimal cough and sputum but gradually increasing effort dyspnoea over two years. Pipe smoker only. FEV $37 \%$ of predicted; TF $15 \%$ predicted; $\mathbf{P a O}_{2}$ $59 \mathrm{mmHg}$.

Radiologist's opinion A bulla in the right upper lobe compressing the right lower lobe which is much reduced in volume; the right middle lobe had reduced vascular markings.

Comparison of radiology and regional function tests (Fig. 14) Again there is good general agreement between the three methods.

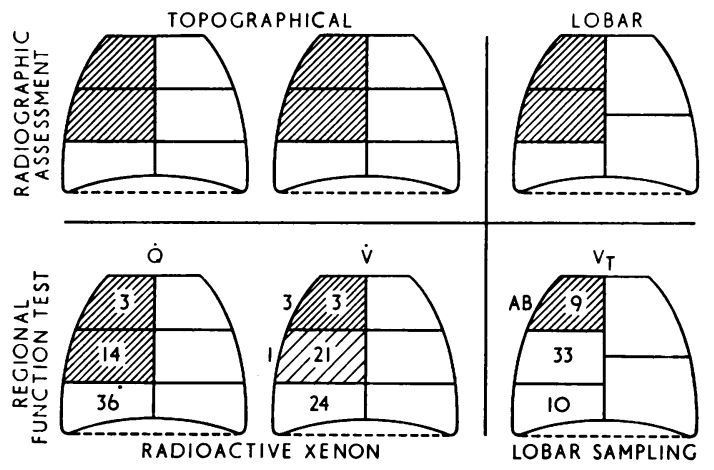

FIG. 14. Results for patient 9.

Thoracotomy Right upper lobe was an air-filled sac (bulla). Remainder of the right lung was also emphysematous.

Comment Again the xenon wash-out was prolonged in the relevant zone and the lobar sampling showed an ' $A$ ' pip in the lobe in which the bulla was situated. 
CASE 10 (D.M., male, aged 41) Winter bronchitis for many years. Progressive effort dyspnoea for seven years following a severe respiratory infection one year previously. Smoked 20 to 30 cigarettes/day. FEV $_{1} 24 \%$ of predicted ; TF $22 \%$ predicted ; $\mathrm{PaO}_{2} 69 \mathrm{mmHg}$.

Radiologist's opinion Widespread emphysema. Bullae were most apparent in the right upper and middle lobes.

Comparison of radiology and regional function tests (Fig. 15) Although the xenon scan for blood flow shows no apparent abnormality, this will occur with widespread disease since this method simply compares one area with another and then becomes uninformative. But within these limits it does agree with the topographical assessment of the radiograph and the somewhat prolonged washout does show that a ventilating abnormality is present. The lobar sampling again agrees with the anatomical radiological assessment in showing the major change in the right middle lobe.

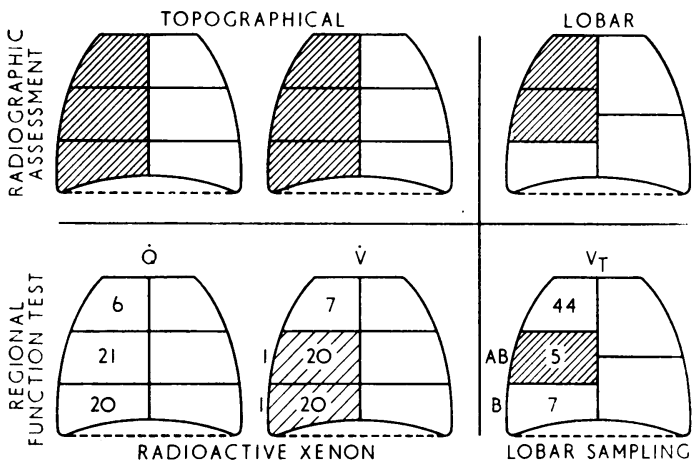

FIG. 15. Results for patient 10.

Thoracotomy Widespread emphysema with bullae most marked in the right middle lobe.

Comment Again the 'A' pip at bronchoscopy was present in the bullous area.

CASE 11 (D.B., male, aged 42 years) Increasing dyspnoea for three years. Quite well before that time. Heavy cigarette smoker with 'smoker's cough' for five years. $\mathrm{FEV}_{1} 49 \%$ of predicted; TF $42 \%$ predicted; $\mathrm{PaO}_{2} 83 \mathrm{mmHg}$.

Radiologist's opinion Large bullae in right upper and middle lobes compressing the right lower lobe.

Comparison of radiology and regional function tests (Fig. 16) The topographical radiological appearances in the PA view in no way reflect the

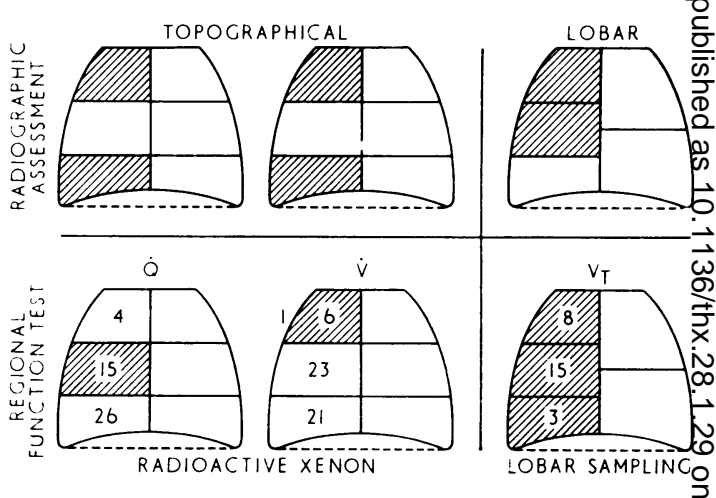

FIG. 16. Results for patient 11 .

true anatomical abnormality. Nor does the xenom scan agree well with the PA view. On the other hand, the lobar sampling does agree with the anatomical radiological findings when one rememo bers that the right lower lobe, although radiologically not abnormal, is compressed and would be expected, therefore, to have reduced ventilas tion.

Thoracotomy Unfortunately a full report wao not available as the operation was done elsewhere but two large bullae and one small one were foun in the right upper lobe.

CASE 12 (P.D., male, aged 48 years) Dyspnoe for 10 years preceded bronchitis which had been present for eight years. $\mathrm{FEV}_{1} 14 \%$ of predicted TF $27 \%$ predicted; $\mathrm{PaO}_{2} 65 \mathrm{mmHg}$.

Radiologist's opinion Bullae in right upper ang middle lobes compressing the right lower lobe.

Comparison of radiology and regional function tests (Fig. 17) There is complete agreement between the three methods, but none suggests that there is any difference in degree of abno

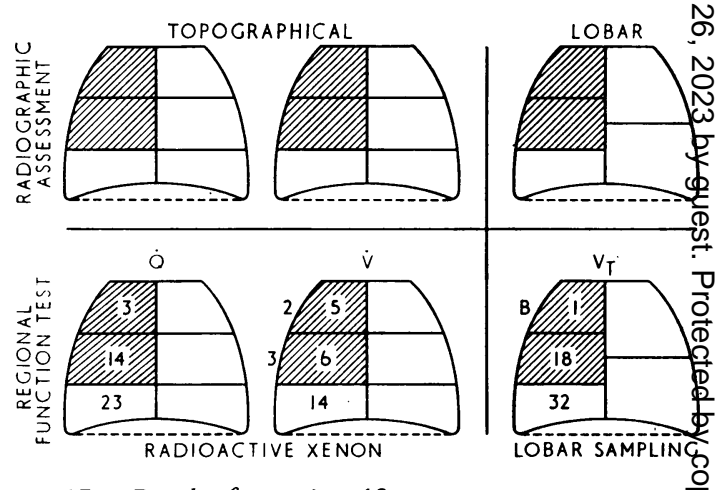

FIG. 17. Results for patient 12. 
mality between the upper and middle lobes except that the 'B' pip at bronchoscopy might suggest that the former was the more abnormal.

Thoracotomy Large bulla in right upper lobe ; right middle and right lower lobes also emphysematous.

COMMENTS ON CASES 7 TO 12 In those patients who had more than one abnormal lobe on radiology, the xenon findings largely agreed with the topographical PA radiological appearances but added nothing to them. On the other hand, the lobar sampling was more useful than the radiograph in four of these six patients in showing, by the presence of ' $A$ ' pips, where the bullae occurred.

\section{DISCUSSION}

Radiographs are often relied on for the localization of emphysema and for inferring functional change; it is important to know when they are adequate for this purpose. The present study shows this to be so when only one lobe appears radiologically abnormal in the lung under consideration. This criterion is fulfilled in cases 1 to 5. All these lesions were grossly bullous on radiography, but we do not know whether nonbullous emphysema can be recognized so well.

Should emphysema be seen in more than one area of the radiograph, however, there is no doubt that regional function tests do help to confirm or refute this radiological diagnosis, even though one lobe seems clearly more abnormal than another.

Bentivoglio et al. (1963) have used xenon-133 scans in diffuse emphysema to show that the most reliable index of regional abnormality is given from the results of radioactive gas wash-in time. Similarly, in our series, when more than one lobe was abnormal on the radiograph, increased xenon wash-out times (which are comparable to wash-in times) provided the best indication of the most abnormal area. This information is topographical, however, and for this reason it gave inadequate lobar localization in most of the six cases with multilobar emphysema. The exceptions are cases 8 and 10 , both of whom had bullae in the right upper lobe; but a large bulla arising from the apex of the right lower lobe might have given identical topographical findings.

Lobar sampling, on the other hand, gives more precise anatomical data (Ritchie and Hugh-Jones, 1966). Asynchronous ventilation has indicated the most abnormal lobe in four of the same six patients (cases $6,7,8$, and 9). The value of this anatomical information is well shown in case 8 . Here the xenon-133 scan merely reproduced the topographical data available from radiography, but lobar sampling correctly identified the right lower lobe as the origin of the bulla. It is clear that the combination of radiographs and lobar sampling is more likely than radiographs paired with lung scanning to demonstrate the site of bullous emphysema. The paradox in case 12 of radiological vascular deficiency in the upper two zones, in contrast to the defect in the lower zone of the xenon-133 scan, may be explained by similar considerations. Lateral films show that the bullous area projects backwards into the lower zone to a greater extent than into the middle and upper zones.

Most work on regional lung function in emphysema has been done using radio-isotopes. Ball et al. (1962) investigated the distribution of ventilation and lung perfusion in emphysematous patients with xenon-133. Nairn, Prime, and Simon (1969) showed that bronchitis caused a more abnormal xenon wash-in time to the lower zone compared to the upper, but this effect was not found to mask the findings in localized emphysema. Shibel, Landis, and Moser (1969), using the xenon-133 scintillation scan, mentioned gaps present in areas where emphysematous bullae were seen on radiographs, but no further correlation was made. In nearly all of these xenon studies, as in ours, the patient was seated upright. We used this position for convenience and so as to be able to compare the xenon with the usual radiographic technique. As in normal subjects seated upright there is diminished blood flow to the lung apices compared with the bases (West, 1970), it may be that some apical vascular defects will have been less apparent than if the patients had been supine. But, from the bronchoscopic studies, for which the patient was supine, we do not believe this factor to have been important in this series of patients.

We conclude that when only one lobe is abnormal on the radiograph this alone is adequate to locate the major site of the emphysema and regional function tests offer relatively little extra information. Occasionally xenon-133 scans give good information about the state of the remaining parts of the lung not available by radiology, but this information can be deduced from a combination of radiology and tests of overall lung function, particularly $\mathrm{Kco}$ and lung compliance measurements (Pride et al., 1970, 1973). Regional sampling is especially helpful in determining if the affected lobe is acting as a ventilating dead 
space. When more than one lobe is affected by emphysema, lobar sampling supplements the radiograph in defining the site of bullous change, as well as locating dead space. Although xenon scans have been helpful for assessing local function in normal lungs (for example, Kaneko et al., 1966) and in many pathological studies, their value in the preoperative assessment of patients with emphysema is limited mainly because the information they provide is topographical. We believe that a combination of radiology and lobar sampling is the best way of assessing which emphysematous patients are likely to benefit from surgery. Xenon scans, although widely employed for this purpose, could well be dispensed with if full radiological studies are made.

We are grateful to the physicians and surgeons who kindly referred patients to us for assessment, and especially to Mr. A. M. Macarthur, F.R.C.S., who operated on most of these patients and told us of his findings. The Medical Research Council generously supported one of us (C. E. B.) during the time he was working on this project.

\section{REFERENCES}

Ball, W. C. J., Stewart, P. B., Newsham, L. G. S., and Bates, D. V. (1962). Regional pulmonary function studies with $\mathrm{Xe}^{133}$. J. clin. Invest., 41, 519.

Bentivoglio, L. G., Beerel, F., Stewart, P. B., Bryan, A. C., Ball, W. C., Jr. and Bates, D. V. (1963). Studies of regional ventilation and perfusion in pulmonary emphysema using xenon ${ }^{133}$. Amer. Rev. resp. Dis., 88, 315.

Dollery, C. T., and Gillam, P. M. S. (1963). The distribution of blood and gas within the lungs measured by scanning after administration of ${ }^{133} \mathrm{Xe}$. Thorax, 18, 316.

Fowler, K. T., and Hugh-Jones, P. (1957). Mass spectrometry applied to clinical practice and research. Brit. med. J., $1,1205$.
Hugh-Jones, P. (1967). Localization of disordered function.ర Bull. Physio-Path. resp., 3, 419.

- Ritchie, B. C., and Dollery, C. T. (1966). Surgical treatment of emphysema. Brit. med. J., 1, 1133.

- , and West, J. B. (1960). Detection of bronchial and arterial obstruction by continuous gas analysis fromes individual lobes and segments of the lung. Thorax, 15, 154.

Hutchison, D. C. S., and Smith, L. A. (1971). Unpublished $\overrightarrow{\vec{\omega}}$ data. Personal communication.

Kaneko, K., Milic-Emili, J., Dolovich, M. B., Dawson, A., and Bates, D. V. (1966). Regional distribution of ventilation and perfusion as a function of body position. $N$ J. appl. Physiol., 21, 767.

Laws, J. W., and Heard, B. E. (1962). Emphysema and the chest film; a retrospective radiological and pathological study. Brit. J. Radiol., 35, 750.

McGrath, M. W., and Hugh-Jones, P. (1963). Some obser-vations on the distribution of gas flow in the human bronchial tree. Clin. Sci., 24, 209.

Nairn, J. R., Prime, F. J., and Simon, G. (1969). Association? between radiological findings and total and regionak function in emphysema. Thorax, 24, 218.

Pride, N. B., Barter, C. E., and Hugh-Jones, P. (1973). Amer. Rev. resp. Dis., in press.

- - Hugh-Jones, P., O'Brien, E. N., and Smith, L. A.O (1970). Changes in lung function following the surgical treatment of bullous emphysema. Quart. J. Med., 39, $49 \overline{\bar{O}}$

Ritchie, B., and Hugh-Jones, P. (1966). Studies of lobarم function in the lungs of patients with emphysema. $\mathbb{Q}$ Scand. J. resp. Dis., Suppl. 62, 83.

Shibel, E. M., Landis, G. A., and Moser, K. M. (1969)음 Inhalation lung scanning evaluation-radioaeroso $P$ versus radioxenon techniques. Dis. Chest, 56, 284.

West, J. B. (1960). Measurement of bronchial air flow J. appl. Physiol., 15, 976. (1970). Ventilation, Blood Flow and Gas Exchange, 2ndo ed. Blackwell, Oxford.

- and Hugh-Jones, P. (1959). Effect of bronchial ando arterial constriction on alveolar gas concentrations in lobe of the dog's lung. J. appl. Physiol., 14, 743. 